\title{
Preterm Birth Is a Novel, Independent Risk Factor for Altered Cardiac Remodeling and Early Heart Failure: Is it Time for a New Cardiomyopathy?
}

\section{Holger Burchert, MSC} Adam J. Lewandowski, DPhil ${ }^{\star}$

\author{
Address \\ *Level 1, John Radcliffe Hospital, 0xford Cardiovascular Clinical Research Facility, \\ Division of Cardiovascular Medicine, Radcliffe Department of Medicine, University \\ of 0xford, 0xford, 0X3 9DU, UK \\ Email: adam.lewandowski@cardiov.ox.ac.uk
}

Published online: 14 February 2019

(C) The Author(s) 2019

This article is part of the Topical Collection on Pediatric and Congenital Heart Disease

Keywords Preterm birth · Cardiomyopathy $\cdot$ Cardiac phenotype $\cdot$ Heart failure $\cdot$ Cardiac remodeling $\cdot$ Hypertension

\section{Abstract}

Purpose of review Around $10 \%$ of the global population is born preterm ( $<37$ weeks' gestation). Preterm birth is associated with an increased risk of cardiovascular events, with preterm-born individuals demonstrating a distinct cardiac phenotype. This review aims to summarize the main phenotypic features of the preterm heart and directions for future research to develop novel intervention strategies.

Recent findings Being born between 28 and 31 weeks' gestation results in a 4-fold higher risk of heart failure in childhood and adolescence and 17 -fold increased risk when born less than 28 weeks' gestation. In support of this being due to a reduction in myocardial functional reserve, preterm-born young adults have an impaired left ventricular cardiac systolic response to moderate and high intensity physiological stress, despite having a preserved resting left ventricular ejection fraction. Similar impairments under physiological stress were also recently reported regarding the right ventricle in young adults born preterm.

Summary Preterm birth relates to a unique cardiac phenotype with an impaired response to stress conditions. These data, combined with the work in animal models, suggest that being born preterm may lead to a novel form of cardiomyopathy. Understanding the driving 
mechanisms leading to this unique cardiac phenotype is important to reduce risk of future heart failure and cardiovascular events.

\section{Introduction}

Globally, the majority of infants born preterm now survive into adulthood due to prenatal and postnatal medical improvements in recent decades. Based on a recently published systematic review and modeling analysis, the estimated global preterm birth rate increased from $9.8 \%$ in the year 2000 to $10.6 \%$ in 2014 , leading to 14.8 million preterm live births in 2014 [1]. The rates in the USA increased for the third year in a row to $9.9 \%$ people born preterm in 2017 [2]. Furthermore, absolute birth numbers continue to rise globally, as do the risk factors leading to preterm birth, meaning the subgroup of individuals born preterm is rapidly increasing, and thus of growing public health relevance.

Besides numerous acute complications, the association from epidemiological data of preterm birth with cardiovascular-related diseases such as stroke, hypertension, atrial fibrillation, and early heart failure is concerning $[3,4 \bullet \bullet, 5]$. Indeed, high blood pressure is already apparent in childhood [6,7] and extends into young adulthood $[8,9]$, which raises concerns about long-term consequences, as high blood pressure is a major independent risk factor for the development of cardiovascular diseases [10]. A systematic review in 2012 summarized that the increase in systolic blood pressure of preterm-born children and young adults was $3.8 \mathrm{mmHg}(95 \% \mathrm{CI}, 2.6-5.0 \mathrm{mmHg})$ higher compared to term-born controls [11], which is in line with findings from a Swedish birth registry cohort including 636,000 individuals where anti-hypertensive medication prescription rates were increased in those born preterm [5]. Moreover, preterm birth is associated with reduced insulin sensitivity [12-14], increased whole-body adiposity, as well as higher intrahepatocellular and intramyocellular lipids [15], which are typical characteristics of the metabolic syndrome. Of mechanistic interest was the finding that increased systolic blood pressure in preterm-born young adults is associated with an enhanced antiangiogenic state in adulthood that seems to be mediated through capillary rarefaction and is independent of other cardiovascular changes [16]. Increases in arterial stiffness have also repeatedly been reported at different stages of development, which may contribute to their increased risk of cardiovascular events in the long term [17-20].

Research to better understand cardiovascular risk has revealed that being born preterm relates to long-term alterations in cardiac structure and function, with a recent finding of an independent risk for early heart failure $[4 \bullet \bullet]$. In this review, we will discuss some of the unique cardiac alterations in the preterm-born population that may make them more susceptible to heart failure and long-term cardiovascular risk.

\section{Adult cardiac phenotype in offspring born preterm}

The first studies to demonstrate long-term alterations in the left and right ventricular phenotypes in preterm-born offspring were performed using cardiac magnetic resonance (CMR) imaging. In a cohort of $n=234$ individuals $(n=102$ born preterm) with follow-up data since recruitment at birth, use of CMR revealed that the mass of the left ventricle of young adults born preterm was on average $19 \mathrm{~g}(\sim 20 \%)$ higher compared to term-born controls and remained significantly higher when indexed to body surface area $[21 \bullet \bullet]$. In addition, the more premature the birth, the greater the mass of the left ventricle in adult life. Though left ventricular mass was positively associated with blood pressure in both preterm-born and term-born participants, preterm-born individuals 
showed additional increases in left ventricular mass independent of higher blood pressure levels. Through creation of a computational atlas of the left ventricle based on CMR [22], geometrical changes were found, comprising shorter ventricles, increased wall thickness, a shift in the apex orientation away from the right ventricle as well as a reduced internal cavity diameter. There were no differences in resting left ventricular ejection fraction or cardiac output, though functional parameters such as left ventricular stroke volume and myocardial deformation patterns [23] were significantly lower in the preterm-born group. In multivariable regression models, the degree of prematurity was the strongest independent predictor of gestational age for structural left ventricular changes, though maternal preeclampsia, which is an antecedent to $\sim 20 \%$ of preterm births and a known risk factor for both the mother and offspring [2431], was also an independent predictor of the lower longitudinal peak systolic strain seen in the preterm-born young adults compared to term-born controls.

Similar findings to the left ventricle were later reported regarding the right

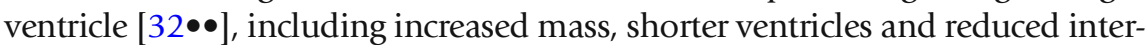
nal cavity diameters. Higher right ventricular mass was also associated with the degree of prematurity, though the relative increase in mass per gestational week was twice as large as previously reported for the left ventricle. In contrast to the left ventricle, right ventricular resting ejection fraction was significantly reduced with $21 \%$ of participants having values below the lower limit observed in the term-born adults and $6 \%$ having mild systolic dysfunction. In multivariable regression models to describe the variation in right ventricular structure and function, the degree of prematurity was the strongest independent predictor of higher right ventricular mass and lower right ventricular end-diastolic volume and ejection fraction. Interestingly, increased number of days on mechanical ventilation in the early postnatal period was also an independent predictor of the increased right ventricular mass.

\section{Insight from animal models and experimental studies}

Studies in animal models have provided important insights into the cardiac phenotype related to preterm birth and potential mechanisms that may underlie these alterations. In a landmark study for the field, Bensley et al. [33•] used an ovine model of preterm birth (14 days premature, equivalent to 36 weeks' gestation in humans) to assess cardiac maturation compared to term-born lambs. The authors demonstrated that the cardiomyocytes of both the left and right ventricles were hypertrophied in the preterm-born lambs compared to controls. Of note was that the preterm-born sheep also had an $\sim 7$-fold increase in interstitial collagen deposition in the lambs born preterm, even without the normal inflammatory and stress-related conditions that are commonly associated with preterm birth in humans [34]. Nucleation and ploidy of the cardiomyocytes were also shown to be altered, which supports the argument that there are irreversible myocardial stress-related changes in DNA in the preterm-born lambs. In line with this, Bertagnolli et al. [35•] showed in a preterm experimental model using rats that prematurity-related stress in the form of hyperoxia could elicit left ventricular remodeling and impaired systolic function prior to blood pressure elevation. These changes included an increase in cardiomyocyte hypertrophy and collagen deposition. Moreover, these early 
cardiac alterations increased susceptibility to developing heart failure secondary to the insult of persistent hemodynamic overload induced by angiotensin II infusion [36•]. Of note, the authors showed that early treatment with the angiotensin II type 1 (AT1) receptor antagonist Losartan prevented the development of cardiac alterations in later life, including fibrosis and hypertrophy. In a similar rat model of preterm-related stress conditions, Goss et al.[37] showed that the hyperoxia-exposed rats had increased right ventricular mass and wall thickness, with a reduced right ventricular ejection fraction, similar to the

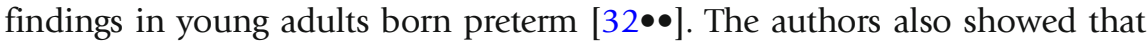
their preterm rat model demonstrated evidence of mitochondrial DNA damage and mitochondrial dysregulation, which may be a contributing factor underlying right ventricular dysfunction seen in young adults born preterm $[32 \bullet \bullet]$.

\section{Key window for emergence of cardiac changes in humans}

Initial studies in infants born preterm provided early insight that alterations in cardiac structure and function might be present as early as the immediate postnatal period and continue to evolve throughout the first year of life [3841]. In a cardiac autopsy study, Bensley et al.[42] compared the cardiomyocyte proliferation of preterm infants with age-matched control fetuses. They demonstrated reduced cardiomyocyte proliferation in preterm infants compared to age-matched stillborn infant controls, possibly caused by the increased arterial pressure and heart rate that occurs during the transition into postnatal life. This reduction in cardiomyocyte proliferation may adversely impact the final number of cardiomyocytes and regenerative capacity of the myocardium, thus reducing the myocardial functional reserve of the preterm heart.

To better understand the cardiac developmental patterns of preterm-born infants from early prenatal through to postnatal life, Aye et al. [43・•] performed echocardiography scans of 392 infants between 15 weeks' gestation and 3 months' postnatal age. By creating trajectories of cardiac development using measures of left and right ventricular mass at different time points during fetal and infant life, it was demonstrated that preterm cardiac changes first emerge in the early postnatal period, with similar in utero cardiac growth trajectories for fetuses that went on to be born preterm or term. Indeed, preterm infants had a disproportionate increase in both left and right ventricular mass that emerged over the first 3 months of life, with an average mass change double that of their term-born counterparts during this period. A reduction in left ventricular diastolic function also emerged with a persistent reduction in right ventricular systolic function. Echocardiography imaging data was used to create computational statistical shape models of the two-dimensional anatomy, which revealed that the cardiac growth changes were not simply a matter of size, but also of cardiac shape remodeling, which may have implications for cardiac flow dynamics and pump efficiency.

Cox et al. [44] recently provided further, detailed insight into the remodeling pattern of the preterm heart during the critical postnatal window using CMR in this population for the first time. Thirty-four preterm infants underwent CMR scans of the left and right ventricle within the first week following delivery (postnatal age 3-7 days) and 29 were scanned again at term-corrected age 
(postnatal age 33-136 days; 37-42 weeks' corrected gestational age). Ten termborn controls underwent the same CMR scan protocol at a single time point (postnatal age 2-7 days). The authors showed that preterm-born individuals had significantly elevated weight-indexed left ventricular mass and enddiastolic volume at term-corrected age. Those born at less than 29 weeks' gestation had a $64 \%$ increase in left ventricular mass at term-corrected age compared to the term-born controls. Furthermore, weight-indexed right ventricular mass and right ventricular end-diastolic volume trended towards being greater in the preterm group at term-corrected age, which reached statistical significance in the cohort born at 29-32 weeks' gestational age. Cox et al. also used the CMR scans to create computational atlases of the left and right ventricles from end-diastolic phases, demonstrating that preterm hearts have a more globular left ventricular shape with more spherical blood pool. Interestingly, the degree of prematurity, requirement for respiratory support greater than $48 \mathrm{~h}$ and the administration of antenatal glucocorticoids were all independently correlated with increased left ventricular wall thickness in the preterm-born infants. Given that left and right ventricular volumes are reduced in those born preterm in childhood and young adulthood $[21 \bullet \bullet, 32 \bullet \bullet, 45,46 \bullet \bullet, 47 \bullet \bullet]$, the current study highlights the need for longitudinal cardiac imaging studies tracking the same individuals over time to better understand the evolution of these remodeling patterns throughout development.

To better understand the immediate clinical significance of these alterations in left ventricular geometry, Choudhry et al. [48] performed a retrospective casecontrol study in a cohort of $n=503$ preterm infants born less than $2000 \mathrm{~g}$ with echocardiography scans. Key predictors of altered left ventricular geometry, including increased left ventricular mass index or increased left ventricular relative wall thickness, were postnatal steroid use and being born small for gestational age. The elevation in left ventricular relative wall thickness was associated with longer hospital stay and increased mortality, with 15/94 $(16 \%)$ with elevated left ventricular relative wall thickness having died compared to $3 / 90$ controls (3.3\%).

\section{Risk of early heart failure and reduced myocardial reserve}

In early 2017, Carr et al. published data from a register-based epidemiological study and found a strong association between being born prior to 32 weeks' gestation and increased risk of incident heart failure in childhood through to young adulthood $[4 \bullet \bullet]$. Of note, being born preterm less than 28 weeks' gestation increased the risk of heart failure 17 -fold and $\sim 4$-fold when born between 28 and 31 weeks' gestation. Nevertheless, the adverse cardiac remodeling is unlikely to be the single cause for why two thirds of heart failure cases in children and young adults recover their cardiac function and that the majority of young people with heart failure in Carr's study were born at term [49]. This supports the idea that familial predisposition or other nonpreterm birth related factors remain the most likely origin of acute heart failure. The preterm cardiac phenotype, however, may reduce the myocardial functional reserve such that these individuals are less able to cope with acute and chronic stressors, reducing 
their ability to recover and pushing them further into a chronic cardiovascular disease state [49].

To test the hypothesis that people born preterm have a reduced myocardial functional reserve, Huckstep et al. [46••] investigated the exercise response of the left ventricle of preterm-born young adults compared to term-born controls. In a cohort of $n=101$ normotensive young adults individuals, of which $n=47$ were born preterm, stress echocardiography was conducted at $40 \%, 60 \%$, and $80 \%$ of peak exercise wattage based on maximal cardiopulmonary exercise testing. In agreement with previous studies, the resting left ventricular ejection fraction did not differ between the groups at rest. However, between 40 and $60 \%$ of maximal exercise intensity there was a 3.7\% decline in left ventricular ejection fraction, leading to a $6.7 \%$ lower left ventricular ejection fraction at $60 \%$ intensity that further declined to $-7.3 \%$ of term-born control values at $80 \%$ intensity. Furthermore, the preterm-born young adults had a 56\% reduced submaximal cardiac output reserve compared to term-born control young adults at $40 \%$ of peak exercise capacity. In addition to being independently associated with the degree of prematurity, left ventricular length and resting peak systolic longitudinal strain predicted left ventricular ejection fraction increase from rest to $60 \%$ exercise intensity in the group of young adults born preterm.

In support of these findings, a recently published study investigated the impact of prematurity on the pulmonary vasculature and right ventricle in adulthood

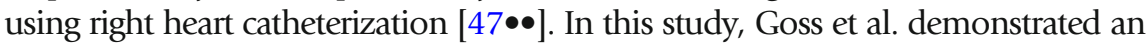
impaired ability of preterm-born young adults to increase right ventricle cardiac index and right ventricular stroke volume during exercise. Moreover, the pretermborn participants showed higher mean pulmonary arterial pressures with $n=3 / 11$ people born preterm meeting criteria for borderline pulmonary hypertension and $n=2 / 11$ meeting the criteria for overt pulmonary hypertension, while none of the term-born group met these criteria.

\section{How can we intervene to prevent cardiovascular risk?}

As indicated by the work of Aye et al. and others, as well as the work from experimental studies, the postnatal developmental window may provide an early opportunity for interventions. Several positive effects on general infant health due to human milk have been reported, such as brain and cognitive development as well as prevention of high blood pressure [50, 51]. With regard to preterm-born individuals, exclusive human milk feeding through the first weeks of postnatal development has shown to correlate with normalization of aspects of the preterm cardiac phenotype in young adulthood towards that of those born at term [52]. In a follow-up study of preterm-born individuals randomized to different milk feeding diets across five UK hospitals between 1982 and 1986, left and right ventricular end-diastolic volume index were found to be increased by 9.79 and $18.2 \%$ respectively in preterm-born young adults fed exclusively human milk compared to exclusively formula fed preterm infants. Moreover, left ventricular and right ventricular stroke volume index were also increased by 9.79 and $22.1 \%$ in the human milk versus formula group. Exclusively formula fed infants also had increased pulmonary artery diameters and increased pulmonary artery to aortic diameter ratios as adults, which may be a sign of increased risk of pulmonary hypertension. Interestingly, 
pulmonary artery differences were closely related to reductions in right ventricular end-diastolic volume index and stroke volume index. The findings provide further support that promotion of human milk during the early developmental window is warranted, though future work is needed to better understand how to optimize feeding, especially for those born at less than 32 weeks' gestation.

Another potential prevention approach for long-term cardiovascular risk reduction in preterm-born offspring is prescribed lifestyle interventions such as diet and exercise throughout later developmental stages, including childhood, adolescence, and young adulthood. One of the challenges with trying to determine the benefits of prescribed aerobic exercise training in younger populations as a preventative approach to cardiovascular disease risk is that most studies in the literature have been done in middle aged and older participants. In light of this, Williamson et al. [53] conducted a systematic review and metaanalysis in 2016 in an adult population in their early 40s to evaluate randomized control trial evidence of cardiovascular benefits of aerobic exercise training. Reductions of $4.40 \mathrm{mmHg}$ systolic blood pressure and $4.17 \mathrm{mmHg}$ diastolic blood pressure were found following intervention, though the effect disappeared within 12 months' follow-up, suggesting that promotion of long-term adherence is needed. Nevertheless, it is known that regular aerobic exercise training positively influences blood lipid profiles, as well as cardiac and vascular remodeling [54]. It has been shown that regular aerobic activity can reduce blood pressure by lowering systemic vascular resistance [55], which may be partially mediated by vascular remodeling, the renin-angiotensin system, as well as the sympathetic nervous system. Given the known alterations in the renin-angiotensin system [56] and unique cardiac phenotype associated with preterm birth, it remains to be determined whether aerobic exercise training would have the same benefits in a population of preterm-born young adults and how this intervention might be used to reduce risk of hypertension and heart failure in these individuals. To explore this, the Trial of Exercise to Prevent HypeRtension in young Adults (TEPHRA) has been designed [57]. This randomized control trial aims to determine the effect of 16 weeks' guided aerobic exercise training on the cardiovascular system of $n=200$ young adults between the ages of 18 and 35 years, including a subgroup of individuals born preterm. Peripheral vascular measures, blood sampling to look at circulating vascular cells and biomarkers, microvascular assessments, echocardiography imaging, physical activity monitoring, and multi-organ magnetic resonance imaging will be conducted to achieve detailed cardiovascular phenotyping. Overall, the results will support the development of future research and expand our understanding of evidence-based management of cardiovascular risk in both pretermborn and term-born young adult populations.

\section{Conclusions}

In conclusion, preterm birth is associated with a unique cardiac phenotype, independent of other pregnancy complications. The altered remodeling pattern of the heart, combined with the demonstrated reduction in myocardial functional reserve in young adulthood, may explain why preterm-born individuals are at increased risk of early heart failure and cardiovascular events in later life. Together, these data suggest that being born preterm is a likely independent risk 
factor for a novel form of cardiomyopathy. Over time, known risk factors of cardiovascular disease in those born preterm, such as elevated blood pressure and adverse alterations in metabolic profile, will likely act as additional negative stimuli on their already compromised cardiac structure and function. In addition, given the known alterations in other end organs in preterm-born individuals, including the brain, liver, and kidneys, further research is needed to better understand the interplay between these multiple organ systems in those born preterm. Given that $10 \%$ of individuals globally are born preterm, these findings are of immediate public health concern and should be taken into clinical consideration, including regular and long-term follow-up of this growing population subgroup.

\section{Compliance with Ethical Standards}

\section{Conflict of Interest}

The authors declare that they have no conflicts of interest.

Human and Animal Rights and Informed Consent

This article does not contain any studies with human or animal subjects performed by any of the authors.

\section{Open Access}

This article is distributed under the terms of the Creative Commons Attribution 4.0 International License (http://creativecommons.org/licenses/by/4.0/), which permits unrestricted use, distribution, and reproduction in any medium, provided you give appropriate credit to the original author(s) and the source, provide a link to the Creative Commons license, and indicate if changes were made.

\section{Publisher's Note}

Springer Nature remains neutral with regard to jurisdictional claims in published maps and institutional affiliations.

\section{References and Recommended Reading}

Papers of particular interest, published recently, have been highlighted as:

- Of importance

-. Of major importance 1. Chawanpaiboon S, Vogel JP, Moller A-B, Lumbiganon
P, Petzold M, Hogan D, Landoulsi S, Jampathong N, Kongwattanakul K, Laopaiboon $\mathrm{M}$ et al: Global, regional, and national estimates of levels of preterm birth in 2014: a systematic review and modelling analysis. Lancet Glob Health 2018.

2. Premature Birth Report Cards | March of Dimes [https://www.marchofdimes.org/mission/prematurityreportcard.aspx].

3. Larsson SC, Drca N, Jensen-Urstad M, Wolk A. Incidence of atrial fibrillation in relation to birth weight and preterm birth. Int J Cardiol. 2015;178:149-52.

4.• Carr H, Cnattingius S, Granath F, Ludvigsson JF, Edstedt Bonamy A-K. Preterm birth and risk of heart failure up to early adulthood. J Am Coll Cardiol. 2017;69(21):2634-42

This large register-based epidemiological study in 2.67 million births in Sweden between 1987 and 2012 demonstrated that 
being born preterm less than 28 weeks' gestation increased the risk of incident heart failure 17 -fold and $\sim 4$-fold when born between 28 and 31 weeks' gestation.

5. Crump C, Winkleby MA, Sundquist K, Sundquist J. Risk of hypertension among young adults who were born preterm: a Swedish national study of 636,000 births. Am J Epidemiol.

2011;173(7):797-803.

6. Bonamy AKE, Martin H, Jörneskog G, Norman M. Lower skin capillary density, normal endothelial function and higher blood pressure in children born preterm. J Intern Med. 2007;262(6):635-42.

7. Bonamy A-KE, Källén K, Norman M. High blood pressure in 2.5-year-old children born extremely preterm. Pediatrics. 2012;129(5):e1199-204.

8. Davis EF, Lewandowski AJ, Aye C, Williamson W, Boardman H, Huang R-C, et al. Clinical cardiovascular risk during young adulthood in offspring of hypertensive pregnancies: insights from a 20-year prospective follow-up birth cohort. BMJ Open.

2015;5(6):e008136.

9. Norman M. Preterm birth—an emerging risk factor for adult hypertension? Semin Perinatol. 2010;34(3):183-7.

10. Lim SS, Vos T, Flaxman AD, Danaei G, Shibuya K, Adair-Rohani $\mathrm{H}$, et al. A comparative risk assessment of burden of disease and injury attributable to 67 risk factors and risk factor clusters in 21 regions, 1990-2010: a systematic analysis for the Global Burden of Disease Study 2010. Lancet. 2012;380(9859):2224-60.

11. de Jong F, Monuteaux MC, van Elburg RM, Gillman MW, Belfort MB. Systematic review and meta-analysis of preterm birth and later systolic blood pressure. Hypertension. 2012;59(2):226-34.

12. Kajantie E, Strang-Karlsson S, Hovi P, Wehkalampi K, Lahti J, Kaseva N, et al. Insulin sensitivity and secretory response in adults born preterm: the Helsinki Study of Very Low Birth Weight Adults. J Clin Endocrinol Metab. 2015;100(1):244-50.

13. Tinnion R, Gillone J, Cheetham T, Embleton N. Preterm birth and subsequent insulin sensitivity: a systematic review. Arch Dis Child. 2014;99(4):362-8.

14. Kelly BA, Lewandowski AJ, Worton SA, Davis EF, Lazdam M, Francis J, et al. Antenatal glucocorticoid exposure and long-term alterations in aortic function and glucose metabolism. Pediatrics.

2012;129(5):e1282.

15. Thomas EL, Parkinson JR, Hyde MJ, Yap IKS, Holmes E, Doré CJ, et al. Aberrant adiposity and ectopic lipid deposition characterize the adult phenotype of the preterm infant. Pediatr Res. 2011;70(5):507-12.

16. Lewandowski AJ, Davis EF, Yu G, Digby JE, Boardman $\mathrm{H}$, Whitworth $\mathrm{P}$, et al. Elevated blood pressure in preterm-born offspring associates with a distinct antiangiogenic state and microvascular abnormalities in adult life. Hypertension. 2015;65(3):607-14.

17. Tauzin L, Rossi P, Grosse C, Boussuges A, Frances $Y$, Tsimaratos $\mathrm{M}$, et al. Increased systemic blood pressure and arterial stiffness in young adults born prematurely. J Dev Orig Health Dis. 2014;5(6):448-52.

18. Peters M, Hoover M, Sturgeon M, Vellookunnel S, Chandrasekar S, Schaeffer GV, Sindler AL, Pierce GL,

Stauss HM, Farrell ET et al: Arterial pulse wave velocity is increased in prematurely-born adults and rats given neonatal supplemental oxygen. FASEB J 2017, 31(1_supplement):1011.1023-1011.1023.

19. Boardman H, Birse K, Davis EF, Whitworth P, Aggarwal $\mathrm{V}$, Lewandowski AJ, et al. Comprehensive multimodality assessment of regional and global arterial structure and function in adults born preterm. Hypertens Res. 2016;39(1):39-45.

20. Lewandowski AJ, Lazdam M, Davis E, Kylintireas I, Diesch J, Francis J, et al. Short-term exposure to exogenous lipids in premature infants and long-term changes in aortic and cardiac function. Arterioscler Thromb Vasc Biol. 2011;31(9):2125-35.

21.• Lewandowski AJ, Augustine D, Lamata P, Davis Esther F, Lazdam M, Francis J, et al. Preterm heart in adult life: cardiovascular magnetic resonance reveals distinct differences in left ventricular mass, geometry, and function. Circulation. 2013;127(2):197-206

This was the first detailed study in humans to demonstrate a unique cardiac phenotype in young adults born preterm.

Using cardiovascular magnetic resonance imaging and computational atlas formation, preterm-born young adults were shown to have an altered left ventricular structure and function.

22. Lamata $P$, Sinclair M, Kerfoot E, Lee A, Crozier A, Blazevic B, Land S, Lewandowski AJ, Barber D, Niederer $S$ et al: An automatic service for the personalization of ventricular cardiac meshes. J R Soc Interface, 11(91):20131023-20,131,023.

23. Augustine D, Lewandowski AJ, Lazdam M, Rai A, Francis J, Myerson S, et al. Global and regional left ventricular myocardial deformation measures by magnetic resonance feature tracking in healthy volunteers: comparison with tagging and relevance of gender. J Cardiovasc Magn Reson. 2013;15(1):8-8.

24. Lewandowski AJ, Leeson P. Preeclampsia, prematurity and cardiovascular health in adult life. Early Hum Dev. 2014;90(11):725-9.

25. Lazdam M, de la Horra A, Diesch J, Kenworthy Y, Davis E, Lewandowski AJ, et al. Unique blood pressure characteristics in mother and offspring after early onset preeclampsia. Hypertension. 2012;60(5):1338-45.

26. Siepmann T, Boardman H, Bilderbeck A, Griffanti L, Kenworthy Y, Zwager C, et al. Long-term cerebral white and gray matter changes after preeclampsia. Neurology. 2017;88(13):1256.

27. Yu Grace Z, Reilly S, Lewandowski AJ, Aye CYL, Simpson LJ, Newton LD, et al. Neonatal microRNA profile determines endothelial function in offspring of hypertensive pregnancies. Hypertension. 2018;72(4):937-45.

28. Yu GZ, Aye CYL, Lewandowski AJ, Davis EF, Khoo CP, Newton L, et al. Al Haj Zen A, Simpson LJ, O'Brien K et al: Association of maternal antiangiogenic profile at 
birth with early postnatal loss of microvascular density in offspring of hypertensive pregnancies. Hypertension. 2016;68(3):749-59.

29. Davis EF, Lazdam M, Lewandowski AJ, Worton SA, Kelly B, Kenworthy Y, et al. Cardiovascular risk factors in children and young adults born to preeclamptic pregnancies: a systematic review. Pediatrics. 2012;129(6):e1552.

30. Davis EF, Newton L, Lewandowski AJ, Lazdam M, Kelly BA, Kyriakou T, et al. Pre-eclampsia and offspring cardiovascular health: mechanistic insights from experimental studies. Clinical science (London, England: 1979). 2012;123(2):53-72.

31. Lazdam M, Davis EF, Lewandowski AJ, Worton SA, Kenworthy Y, Kelly B, et al. Prevention of vascular dysfunction after preeclampsia: a potential long-term outcome measure and an emerging goal for treatment. J Pregnancy. 2012;2012:704146-704,146.

32.• Lewandowski AJ, Bradlow WM, Augustine D, Davis EF, Francis J, Singhal A, et al. Right ventricular systolic dysfunction in young adults born preterm. Circulation. 2013;128(7):713-20

Using cardiovascular magnetic resonance imaging, this study showed that changes in the right ventricle in preterm-born young adults were even greater than in the left ventricle, with $6 \%$ of those born preterm with clinically significant reductions in right ventricular systolic function.

33. Bensley JG, Stacy VK, De Matteo R, Harding R, Black MJ. Cardiac remodelling as a result of pre-term birth: implications for future cardiovascular disease. Eur Heart J. 2010;31(16):2058-66

In this landmark study in a preterm-born ovine model, the authors demonstrate that lambs born preterm have increased myocardial collagen deposition and increased cardiomyocyte hypertrophy, with additional signs of altered cardiac maturation.

34. Mohamed A, Leeson P, Lewandowski AJ. Like sheep, like humans? Right ventricular remodelling in a preterm-born ovine model. J Physiol.

2018;596(23):5505-6.

35. Bertagnolli M, Huyard F, Cloutier A, Anstey Z, HuotMarchand J-É, Fallaha C, et al. Transient neonatal high oxygen exposure leads to early adult cardiac dysfunction, remodeling, and activation of the reninangiotensin system. Hypertension. 2014;63(1):143-50 In a preterm experimental model using rats, the authors demonstrated that prematurity-related stress in the form of hyperoxia could elicit left ventricular remodeling and impaired systolic function prior to blood pressure elevation. These changes included an increase in cardiomyocyte hypertrophy and collagen deposition.

36. Bertagnolli M, Dios A, Béland-Bonenfant S, Gascon G, Sutherland M, Lukaszewski M-A, et al. Activation of the cardiac renin-angiotensin system in high oxygenexposed newborn rats: angiotensin receptor blockade prevents the developmental programming of cardiac dysfunction. Hypertension. 2016;67(4):774-82

This study demonstrated that early treatment with the angiotensin II type 1 (AT1) receptor antagonist Losartan prevented the development of cardiac fibrosis and hypertrophy in later life in a preterm rat experimental model.

37. Goss KN, Kumari S, Tetri LH, Barton G, Braun RK, Hacker TA, et al. Postnatal hyperoxia exposure durably impairs right ventricular function and mitochondrial biogenesis. Am J Respir Cell Mol Biol. 2017;56(5):609-19.

38. Mikkola K, Leipälä J, Boldt T, Fellman V. Fetal growth restriction in preterm infants and cardiovascular function at five years of age. J Pediatr. 2007;151(5):494499.e492.

39. Kozák-Bárány A, Jokinen E, Saraste M, Tuominen J, Välimäki I. Development of left ventricular systolic and diastolic function in preterm infants during the first month of life: a prospective follow-up study. J Pediatr. 2012;139(4):539-45.

40. Levy PT, Patel MD, Choudhry S, Hamvas A, Singh GK. Evidence of echocardiographic markers of pulmonary vascular disease in asymptomatic infants born preterm at one year of age. J Pediatr. 2018;197:48-56.e42.

41. Levy PT, El-Khuffash A, Patel MD, Breatnach CR, James AT, Sanchez AA, et al. Maturational patterns of systolic ventricular deformation mechanics by twodimensional speckle-tracking echocardiography in preterm infants over the first year of age. J Am Soc Echocardiogr. 2017;30(7):685-698.e681.

42. Bensley JG, Moore L, De Matteo R, Harding R, Black MJ. Impact of preterm birth on the developing myocardium of the neonate. Pediatr Res. 2018;83(4):880-8.

43.• Aye CYL, Lewandowski AJ, Lamata P, Upton R, Davis E, Ohuma EO, et al. Disproportionate cardiac hypertrophy during early postnatal development in infants born preterm. Pediatr Res. 2017;82(1):36-4.

By creating trajectories of cardiac development using measures of left and right ventricular mass measured using echocardiography at different time points during fetal and infant life, it was demonstrated that cardiac changes first emerge in the early postnatal period, with similar in utero cardiac growth trajectories for fetuses that went on to be born preterm or term.

44. Cox DJ, Bai W, Price AN, Edwards AD, Rueckert D, Groves AM: Ventricular remodeling in preterm infants: computational cardiac magnetic resonance atlasing shows significant early remodeling of the left ventricle. Pediatr Res 2018. https://www.nature.com/articles/ s41390-018-0171-0.

45. Mohlkert L-A, Hallberg J, Broberg O, Rydberg A, Halvorsen CP, Liuba P, et al. The preterm heart in childhood: left ventricular structure, geometry, and function assessed by echocardiography in 6-year-old survivors of periviable births. J Am Heart Assoc. 2018;7(2):e007742.

46.• Huckstep OJ, Williamson W, Telles F, Burchert H, Bertagnolli M, Herdman C, et al. Physiological stress elicits impaired left ventricular function in pretermborn adults. J Am Coll Cardiol. 2018;71(12):1347-56 Using echocardiography imaging at prescribed exercise intensities, this study demonstrated that preterm-born young adults have impaired left ventricular functional response to physical 
exercise, which suggests that they have a reduced myocardial functional reserve.

47.• Goss KN, Beshish AG, Barton GP, Haraldsdottir K, Levin TS, Tetri LH, Battiola TJ, Mulchrone AM, Pegelow $\mathrm{DF}$, Palta $\mathrm{M}$ et al: Early pulmonary vascular disease in young adults born preterm. Am J Respir Crit Care Med 2018. https://www.ncbi.nlm.nih.gov/pubmed/ 29944842.

Using right heart catheterization, the authors demonstrated that young adults born preterm were significantly less able to augment cardiac index or right ventricular stroke work during exercise, providing further evidence of a reduced myocardial functional reserve.

48. Choudhry S, Salter A, Cunningham TW, Levy PT, Hackett BP, Singh GK, et al. Risk factors and prognostic significance of altered left ventricular geometry in preterm infants. J Perinatol. 2018;38(5):543-9.

49. Leeson P, Lewandowski AJ. A new risk factor for early heart failure: preterm birth. J Am Coll Cardiol. 2017;69(21):2643-5.

50. Blesa M, Sullivan G, Anblagan D, Telford EJ, Quigley AJ, Sparrow SA, et al. Early breast milk exposure modifies brain connectivity in preterm infants.

NeuroImage. 2019;184:431-9.

51. Singhal A, Cole TJ, Lucas A. Early nutrition in preterm infants and later blood pressure: two cohorts after randomised trials. Lancet.

2001;357(9254):413-9.
52. Lewandowski AJ, Lamata P, Francis JM, Piechnik SK, Ferreira VM, Boardman H, Neubauer S, Singhal A, Leeson P, Lucas A. Breast Milk Consumption in Preterm Neonates and Cardiac Shape in Adulthood. Pediatrics 2016; 138(1).

53. Williamson W, Foster C, Reid H, Kelly P, Lewandowski AJ, Boardman $\mathrm{H}$, et al. Will exercise advice be sufficient for treatment of young adults with prehypertension and hypertension? A systematic review and meta-analysis. Hypertension. 2016;68(1):78-87.

54. Buttar HS, Li T, Ravi N. Prevention of cardiovascular diseases: Role of exercise, dietary interventions, obesity and smoking cessation. Exp Clin Cardiol. 2005;10(4):229-49.

55. Cornelissen VA, Fagard RH. Effects of endurance training on blood pressure, blood pressure-regulating mechanisms, and cardiovascular risk factors. Hypertension. 2005;46(4):667-75.

56. Bertagnolli M. Preterm birth and renin-angiotensinaldosterone system: evidences of activation and impact on chronic cardiovascular disease risks. Protein Pept Lett. 2017;24(9):793-8.

57. Williamson W, Huckstep OJ, Frangou E, Mohamed A, Tan C, Alsharqi $M$, et al. Trial of exercise to prevent HypeRtension in young adults (TEPHRA) a randomized controlled trial: study protocol. BMC Cardiovasc Disord. 2018;18(1):208. 